\title{
Magnetic Fields and Cosmic Rays in Galaxy Clusters
}

\author{
Klaus Dolag \\ MPI for Astrophysic, Karl-Schwarzchild-Str. 1, D-85741 Garching, Germany.
}

\begin{abstract}
In galaxy clusters, non-thermal components such as magnetic field and high energy particles keep a record of the processes acting since early times till now. These components play key roles by controlling transport processes inside the cluster atmosphere and beyond and therefore have to be understood in detail by means of numerical simulations. The complexity of the intra cluster medium revealed by multi-frequency observations demonstrates that a variety of physical processes are in action and must be included properly to produce accurate and realistic models. Confronting the predictions of numerical simulations with observations allows us to validate different scenarios about origin and evolution of large scale magnetic fields and to investigate their role in transport and acceleration processes of cosmic rays.
\end{abstract}

Keywords. magnetic fields, methods: numerical, galaxies: clusters: general

\section{Introduction}

Magnetic fields have been detected in galaxy clusters by radio observations, via the Faraday rotation signal of the magnetized cluster atmosphere towards polarized radio sources in or behind clusters (see Carilli \& Taylor 2002 for a recent review) and from diffuse synchrotron emission of the cluster atmosphere (see Govoni \& Feretti 2004; Ferrari et al. 2008, for recent reviews). However, our understanding of their origin is still very limited. Furthermore, the origin and the evolution of the population of cosmic rays within galaxy clusters are tightly connected to the dynamics of the system and to the evolution of the magnetic field. Therefore, cosmological MHD simulations are a valuable tool to investigate and distinguish different scenarios. See Dolag et al. 2008 for a recent review and figure 1 for a typical simulation network used to simulate cosmological, large scale structures including non-thermal effects.

\section{Radio Emission}

The diffuse radio emission within galaxy clusters is produced by synchrotron radiation of relativistic electrons with the cluster magnetic fields. Such diffuse emission - often refered to as giant radio haloes - is detected over regions spanning $\mathrm{Mpc}$ in size. One basic problem in explaining this phenomena is that the cooling time of such relativistic electrons is much shorter than their diffusion time over the region of interest. Therefore they basically have to be produced locally within the whole radio emitting region. One, often discussed mechanism to produce such relativistic electrons is the so called secondary model, where the relativistic electrons are a product by scattering of cosmic ray protons with thermal protons. Cosmic ray protons can for example be produced within accretion shocks and then advected into the cluster, or directly produced within merger shocks. Due to their larger mass compared to the cosmic ray electrons they can diffuse throughout the radio emitting region within the galaxy cluster without undergoing significant energy losses. Although the scaling between radio luminosity and temperature of the simulated clusters agree well with the observed ones, there are no indications that simulations would be able to produce the class of galaxy clusters, for which no radio emission is observed. In fact, the scatter in the predicted scaling relation is very small, as also found in previous studies (Dolag \& Ensslin 2000, Miniatti et al. 2001, Pfrommer et al. 2007). Even cluster which are undergoing two major merger events lead to very elongated loops along the scaling relation which can not bridge the gap between the clusters with and without observed radio emission 


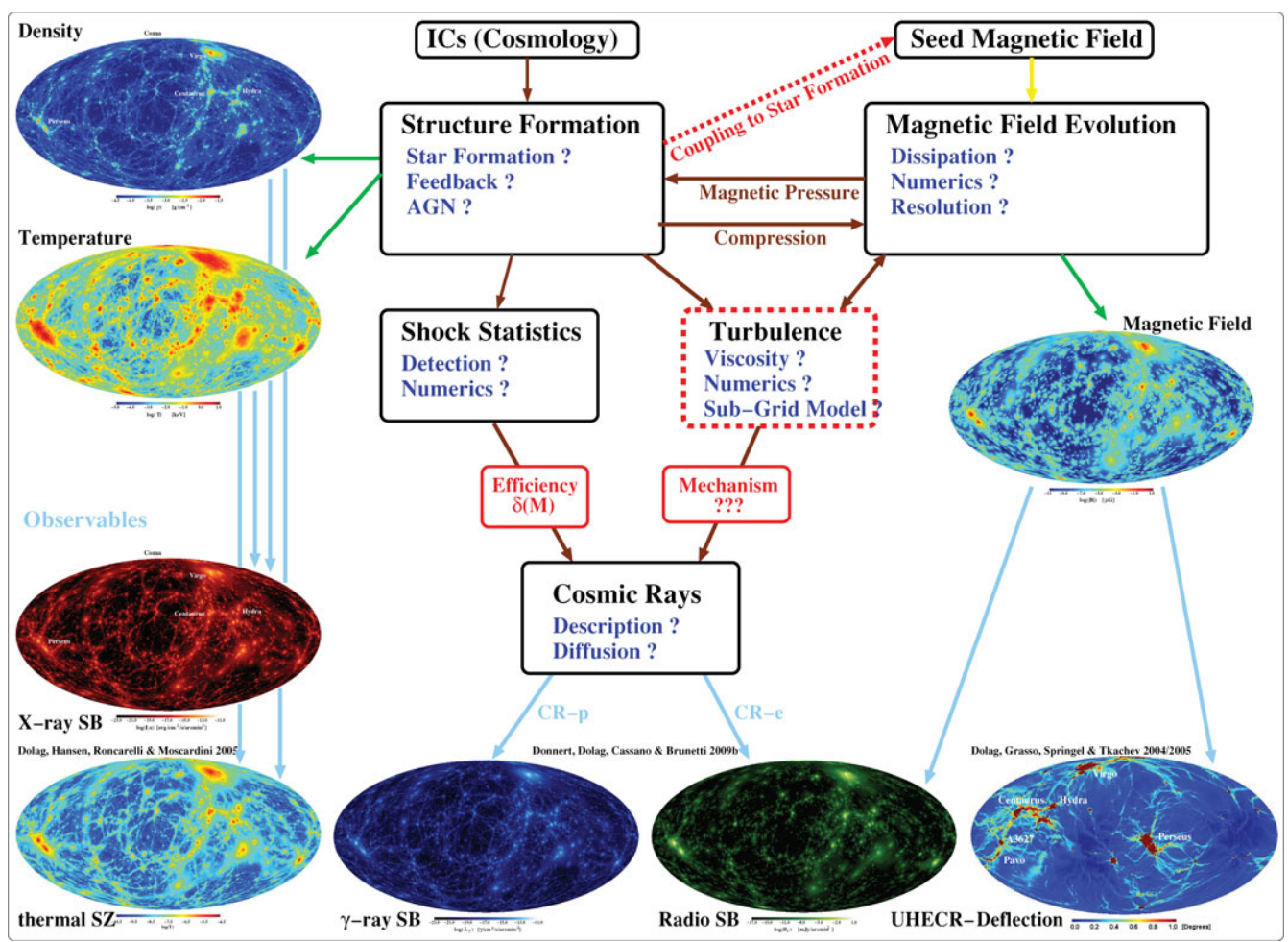

Figure 1. typical simulation network used to simulate cosmological, large scale structures including non-thermal effects.

(see Donnert et al 2009c). Furthermore, such models can not reproduce the observed spectrum of the radio emission for cluster like coma (see Donnert et al. 2009b).

\section{Conclusions}

The increasing amount of available radio data - both, for rotation measures as well as for diffuse radio emission - are driving our understanding of magnetic fields and cosmic rays in galaxy clusters. The improvements in the interpretations of these data over the last years are revealing a quite complex structure of the magnetic fields within galaxy clusters. Also the improvements in the numerical methods are producing more robust predictions for the magnetic field in galaxy clusters, which are helping to interpret the observations. Therefore, in the last years, a consistent picture of the magnetic fields in clusters of galaxies has been emerged from both, numerical work and observations.

Simulations of individual processes like shear flows, shock/bubble interactions or turbulence/merging events predict consistently a super-adiabatic amplification of magnetic fields within such processes. This now has been largely confirmed through direct cluster simulations within a cosmological context. It is worth mentioning that this common result is obtained by using a variety of different codes (see Dolag \& Stassyszyn 2009), which are based on different numerical schemes. Within this context, various observational aspects are reproduced. Moreover, the overall amount of amplification of the magnetic field driven by the structure formation process lead to a final magnetic field strength at a level, sufficient to link models that predict magnetic field seed by various different processes with the magnetic fields observed in galaxy clusters. In fact, the imprint of structure formation onto the magnetic field within galaxy clusters is such strong, that no measurable properties of the initial magnetic seed fields remain inside galaxy clusters. Therefore the only place we can hope to still find signs of the original process of magnetization are mildly non linear regimes of structure formation like filaments (Dolag et al. 1999,2002/2005, Donnert et al. 2009a). 
Such models of magnetic field in galaxy clusters allow also to constrain the origin of cosmic rays within galaxy clusters when confronted with observations of the diffuse radio emission. Although so called secondary models are able to produce sufficient radio emission, a detailed comparison shows that they fail to produce some key observational aspects. Most striking they overproduce the number of galaxy clusters which are expected to show radio emission as well as the fail to produce the observed spectral shape for the diffuse radio emission (see Dolag \& Ensslin 2000, Donnert et al. 2009b/2009c). All this demonstrate the power of such cosmological MHD simulations to learn more about non thermal components like magnetic fields and cosmic rays within galaxy clusters and the large scale structure.

\section{Acknowledgments}

KD acknowledge supported by the DFG cluster of excellence "Origin and Structure of the Universe".

\section{References}

Carilli, C. T. \& Taylor, G. B. 2002, ARAA, 40, 319

Clarke, T. E., Kronberg, P. P., \& Boehringer, H. ApJ, 547, L111

Dolag, K., Bykov, A. M., \& Diaferio, A. 2008, SSRev, 134, 311

Dolag, K., Bratelmann, M., \& Lesch, H. 1999, A\&A, 348, 351

Dolag, K., Bratelmann, M., \& Lesch, H. 2002, A\&A A, 387, 383

Dolag, K. \& Ensslin, T. 2000 A\&A, 362, 151

Dolag, K., Grasso, D., Springel, V., \& Tkachev, I. 2005, Journal of Cosmology and Astro-Particle Physics, 1, 9

Dolag, K. \& Stasyszyn, F. 2009, MNRAS, 398, 1678

Donnert, R., Dolag K., Lesch, H., \& Mueller, E. 2009, MNRAS, 292, 1008

Donnert, R., Dolag K., Cassano, R., \& Brunetti, G., MNRAS, in press

Donnert, R., Dolag K., Brunetti, G., \& Cassano, R., in prep

Ferrari, C., Govoni, F., Schindler, S., Bykov, A. M., \& Rephaeli, Y. 2008, SSRev, 134, 93

Govoni, F. \& Feretti, L. 2004, International Journal of Modern Physics D, 13, 1549

Miniatti, F., Johns, T. W., Kang, H., \& Ryu, D. 2001, ApJ, 512, 233

Pfrommer, C., Ensslin, T. A., Jubelgas, M., Springel, \& Dolag, K. 2007 MNTAS, 378, 385 